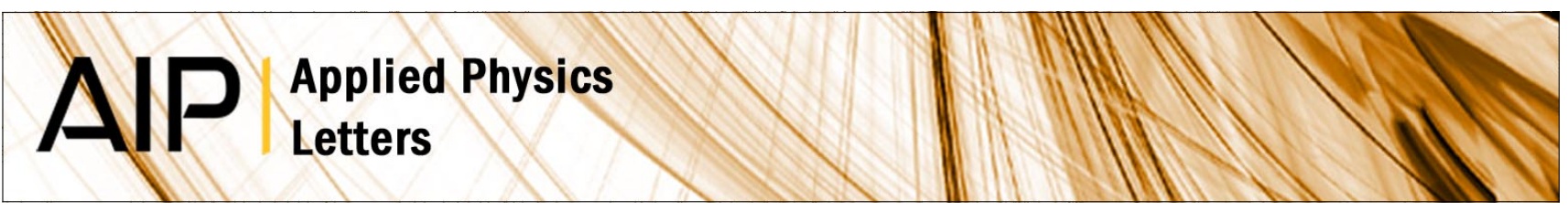

\title{
Broadband photonic control for dual-mode terahertz laser emission
}

Owen P. Marshall, Md. Khairuzzaman, Harvey E. Beere, David A. Ritchie, and Subhasish Chakraborty

Citation: Appl. Phys. Lett. 102, 181106 (2013); doi: 10.1063/1.4804674

View online: http://dx.doi.org/10.1063/1.4804674

View Table of Contents: http://apl.aip.org/resource/1/APPLAB/v102/i18

Published by the American Institute of Physics.

Additional information on Appl. Phys. Lett.

Journal Homepage: http://apl.aip.org/

Journal Information: http://apl.aip.org/about/about_the_journal

Top downloads: http://apl.aip.org/features/most_downloaded

Information for Authors: http://apl.aip.org/authors

\section{ADVERTISEMENT}

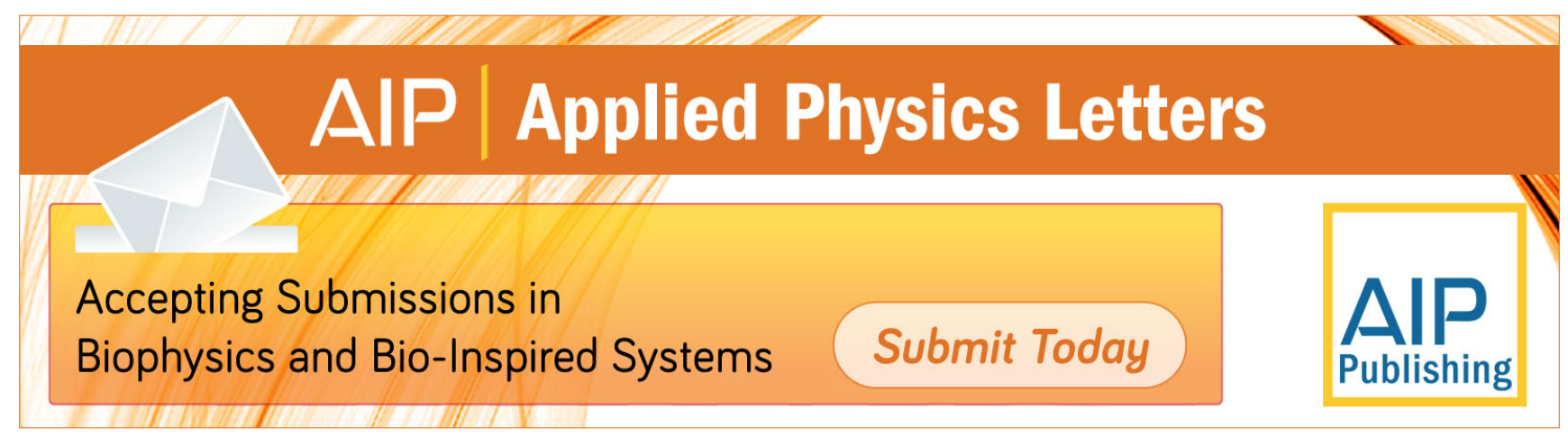




\title{
Broadband photonic control for dual-mode terahertz laser emission
}

\author{
Owen P. Marshall, ${ }^{1}$ Md. Khairuzzaman, ${ }^{1}$ Harvey E. Beere, ${ }^{2}$ David A. Ritchie, ${ }^{2}$ \\ and Subhasish Chakraborty ${ }^{1, a)}$ \\ ${ }^{1}$ School of Electrical and Electronic Engineering, University of Manchester, Manchester M13 9PL, \\ United Kingdom \\ ${ }^{2}$ Cavendish Laboratory, University of Cambridge, JJ Thomson Avenue, Cambridge CB3 OHE, United Kingdom
}

(Received 7 April 2013; accepted 27 April 2013; published online 8 May 2013)

\begin{abstract}
Short, holographically designed, aperiodic distributed feedback (ADFB) gratings are able to provide multi-band spectral filtering over arbitrarily wide bandwidths, offering a complimentary photonic technology to ultra-broadband terahertz quantum cascade lasers (THz QCLs). Using an ADFB grating, ion milled directly into the laser waveguide, high resolution spectral filtering is achieved in $\mathrm{THz}$ QCLs with heterogeneous active regions producing two distinct spectral gain peaks centred around 2.65 and 2.9 THz. Simultaneous dual-mode emission is achieved from a single section laser, with up to 20-dB side-mode suppression. Discrete electronic mode tuning occurs between ADFB bands, giving a switchable mode separation ranging from 163 to $267 \mathrm{GHz}$, along with continuous electronic and thermal tuning of up to $2 \mathrm{GHz}$. (C) 2013 AIP Publishing LLC.
\end{abstract}

[http://dx.doi.org/10.1063/1.4804674]

Terahertz quantum cascade lasers (THz QCLs) are compact, electrically driven, high output power semiconductor devices. ${ }^{1,2}$ Consequently, these laser sources are particularly appealing for $\mathrm{THz}$ applications including imaging, spectroscopy, and high speed data transmission, in sectors as diverse as medicine, security, and telecommunications. ${ }^{3}$ For many applications, a broad emission bandwidth is highly desirable. For example, spectroscopic applications would benefit from increased spectral coverage. This has been a motivating factor in the design of QCLs with ever broader spectral gain. Single active regions generally produce emission spanning a few hundred gigahertz, ${ }^{4}$ but this has been extended to over $1 \mathrm{THz}$ by careful design. ${ }^{5}$ Heterogeneous structures offer an alternative approach to extending the spectral range, stacking multiple active regions within the same laser waveguide ${ }^{6-9}$ following the method established in earlier mid-infrared QCLs. ${ }^{10}$ In this way, ultra-broadband QCL emission has been demonstrated around a centre frequency of $2.7 \mathrm{THz}$, again spanning over $1 \mathrm{THz}^{8}$ There also exist single active region designs providing dual, electronically switchable gain peaks. ${ }^{11,12}$ However, for many practical THz QCL applications, active region design alone is insufficient. A complimentary photonic technology is also required: one that harnesses the broadband optical gain, precisely and dynamically defining the final lasing frequencies. There exist a number of approaches based on external cavities ${ }^{13}$ and gratings, ${ }^{14}$ or mechanically reconfigurable waveguides. ${ }^{15}$ However, these methods involve moving optics or device components, adding undesirable complexity to the final system. An alternative approach, based on electronic control and an integrated optical filter (illustrated schematically in Fig. 1(a)), is therefore preferable. Finely spaced, userdesigned, multi-band optical filters with a spectral response covering such a large bandwidth are non-trivial to produce. Periodic distributed feedback (DFB) gratings have an inherently narrow spectral response (Fig. 1(b)). Although they

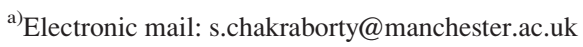

have been used to achieve single emission frequency, ${ }^{16-18}$ the lack of significant refractive index tuning in THz QCLs ${ }^{19}$ means DFB gratings are of little use in defining a broad range of frequencies. Placing a series of DFB gratings of differing periodicities within the same $\mathrm{QCL}^{20}$ is not practical at these operating wavelengths due to the required device size and the difficulty associated with balancing the coupling strengths (and hence the mode selection process) in multiple gratings simultaneously. Quasi-periodic gratings based on fixed mathematical sequences can produce dual-frequency lasing $^{21}$ but offer no means to define and control multiple reflection bands within the gain bandwidth of a $\mathrm{THz}$ QCL. On the other hand, concatenated gratings with an aperiodic basis would require total device lengths of centimetres to generate closely spaced bands at $\mathrm{THz}$ frequencies, which would consume impractical levels of electrical power. ${ }^{22}$ These issues can be avoided by using holographically designed aperiodic distributed feedback (ADFB) gratings. ${ }^{23-25}$ Following computer-generated hologram principles, it is possible to inversely design an ADFB grating to provide a multi-band filter response. ${ }^{24}$ One of a number of advantages to this approach is that filtering bands can be generated across arbitrarily broad bandwidths using a short ADFB structure. A broad, dual-peaked spectral gain $\mathrm{G}(f)$ and an idealised ADFB reflectivity response $\rho(f)$ are illustrated in Figs. 1(c) and 1(d), respectively. The total ADFB bandwidth $(\Omega)$ is large, covering the entirety of $\mathrm{G}(f)$, but individual filtering bands within $\Omega$ are finely spaced. ${ }^{24,25}$ The goal is to choose a target $\rho(f)$ which can work hand in hand with $\mathrm{G}(f)$ for precise mode selection and switching. In this work, $\rho(f)$ contains a series of equally spaced spectral bands of similar strength. This design was previously combined with a singlepeaked gain region for discrete single mode tuning over a frequency span of $>160 \mathrm{GHz} .{ }^{24}$ Essentially, the architecture described in Fig. 1 provides one-to-one mapping between the filtering bands and lasing modes. Here, the same tuning architecture is employed with a heterogeneous active region providing dual-peaked $\mathrm{THz}$ gain. Prior multi-mode lasing is 
(a)

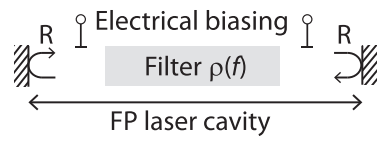

(c) $\mathrm{G}(f) \uparrow$

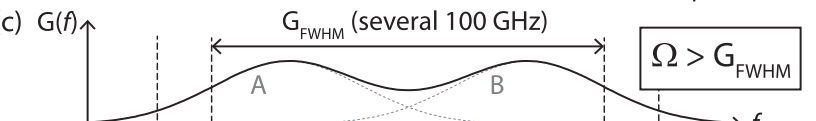

(d)

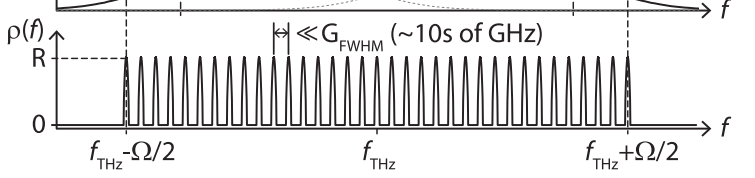

FIG. 1. (a) Schematic tuning architecture, consisting of an electrically biased QCL cavity with an integrated optical filter. (b) Reflectivity response of a periodic DFB grating. (c) Multi-peaked or broadband gain can be achieved in a heterogeneous THz QCL by stacking multiple active regions (i.e., total gain $=\mathrm{A}+\mathrm{B}$ ). (d) A single $\mathrm{ADFB}$ grating can provide multiple, finely spaced filtering bands across an arbitrarily wide bandwidth $(\Omega)$, covering the entire gain width $\left(\mathrm{G}_{\mathrm{FWHM}}\right)$.

suppressed; emission instead occurring on just two ADFB controlled modes, one within each gain peak. By altering the electrical driving conditions, the dual-mode QCL emission may be switched between ADFB bands. The dual-mode frequency separation $\Delta f$ thereby ranges from 163 to $267 \mathrm{GHz}$. Furthermore, continuous electronic and thermal fine tuning of up to $\sim 2.5 \mathrm{GHz}$ is observed for individual modes, enabling $\sim 2 \mathrm{GHz}$ variation in $\Delta f$.

Devices were fabricated from a single molecular beam epitaxially grown $\mathrm{GaAs} / \mathrm{Al}_{0.15} \mathrm{Ga}_{0.85}$ wafer, with two heterogeneous bound-to-continuum active regions (ARs) simultaneously providing gain at $\sim 2.65$ and $2.9 \mathrm{THz}{ }^{9}$ Semi-insulating surface plasmon (SI-SP) waveguides $(160 \mu \mathrm{m}$ wide) were defined by wet chemical etching and thermal evaporation of metals (100 nm PdGe ohmic contact, 20/80 nm Ti/Au overlayer), then cleaved to form $\sim 6 \mathrm{~mm}$ long Fabry-Pérot (FP) cavities. After heatsink packaging and gold wire bonding, QCLs were mounted on the cold-finger of a Janis ST-100 continuous-flow helium cryostat for electrical and optical testing. All testing was performed in pulsed operation at liquid helium temperatures, with a pulse rate of $10 \mathrm{kHz}$ and duty cycles of $\leq 5 \%$. A Bruker Vertex 80 Fourier transform infrared (FTIR) spectrometer was used in conjunction with a QMC helium-cooled bolometric detector to collect spectra with a resolution of $2.25 \mathrm{GHz}\left(0.075 \mathrm{~cm}^{-1}\right)$ in a nitrogen purged environment. Output powers were measured without collection optics using a calibrated large area thermopile detector placed inside the cryostat. An FEI Nova Nanolab 600 focused ion beam (FIB) system $(30 \mathrm{keV}$ Ga ions, $50 \mathrm{~nm}$ nominal spot diameter, $1 \mathrm{nA}$ beam current) was used to mill the ADFB pattern into the upper layers (metal and $n^{+}-\mathrm{GaAs}$ ) of the SI-SP waveguides as a series of narrow, subwavelength slits $(\sim 0.5 \mu \mathrm{m}$ by $100 \mu \mathrm{m})$. Careful control of the FIB process gave a high degree of reproducibility in slit dimensions, including the target depth (the interface between the AR and upper $\mathrm{n}^{+}$-GaAs contact layer). The ADFB pattern was reported in Ref. 24.

In order to discern the influence of $\rho(f)$ on QCL emission, ADFB gratings were introduced to a number of precharacterised devices. Figure 2(a) shows the calculated $\rho(f)$ in the spectral region of interest for the ADFB grating applied to the first device, QCL1. An approximate Fourier

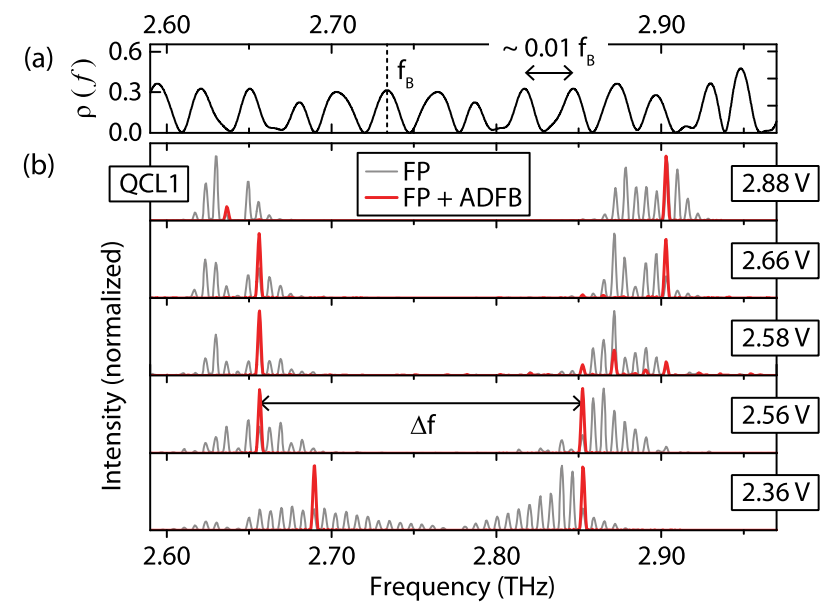

(c)

(d)
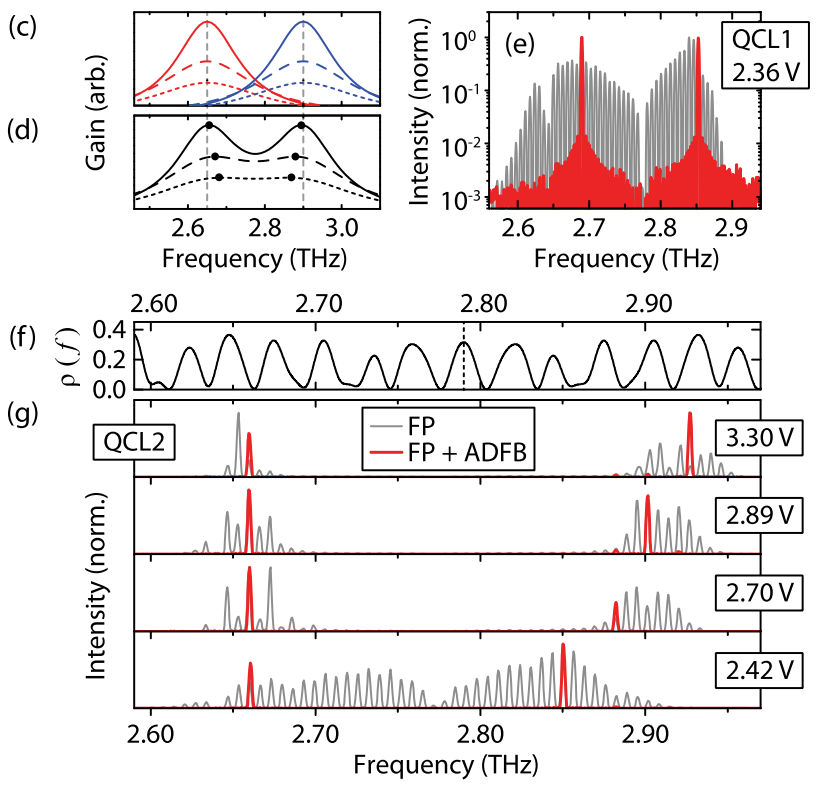

FIG. 2. (a) Calculated reflectivity response of the ADFB structure in QCL1, using $\Lambda=14.9 \mu \mathrm{m}, n_{\mathrm{eff}}=3.68$, and $|\Delta n|=0.1$. (b) Selected emission spectra from QCL1 before (light lines) and after (heavy lines) introduction of the ADFB grating. (c) Illustration of two overlapping but narrowing gain peaks (moving from dotted to solid lines). (d) The corresponding linear sums of the two peaks in (c). (e) In contrast to the original FP spectra, the ADFB selected modes (heavy lines) can possess an SMSR in excess of $20 \mathrm{~dB}$. (f) Calculated reflectivity response of the ADFB structure in QCL2, using $\Lambda=14.6 \mu \mathrm{m}, n_{\text {eff }}=3.68$, and $|\Delta n|=0.1$. (g) Selected emission spectra from QCL2 before (light lines) and after (heavy lines) introduction of the ADFB grating.

transform equation was used to calculate $\rho(f) .{ }^{23-25}$ The complex grating refractive index contrast was assumed to be $|\Delta n|=0.1$, although its true value is not precisely known. A minimum grating element separation $\Lambda=14.90 \mu \mathrm{m}$ was chosen, giving a central $\rho(f)$ band frequency $f_{\mathrm{B}}=2.73 \mathrm{THz}$, assuming an effective refractive index $n_{\text {eff }}=3.68 .^{24}$ The lighter lines in Fig. 2(b) show the FP emission from QCL1 (i.e., prior to the introduction of the ADFB), at selected operating biases. The spectral envelope of the laser emission displays two diverging peaks. This is evidence of biasdependent narrowing in two gain peaks centred around $\sim 2.65$ and $2.9 \mathrm{THz}, 9,26$ as illustrated in Figs. 2(c) and 2(d), which respectively show two narrowing gain peaks with a spectral overlap, and the total effective gain-their linear sum. The effective gain spectra contain tunable maxima (dots), despite the static nature of the two underlying gain 
centre frequencies. Under almost all operating conditions, the FP QCL lased on multiple modes under each gain maxima, with a mode spacing of $\sim 6.5 \mathrm{GHz}$ dictated by the $6.03 \mathrm{~mm}$ cavity length and a group refractive index of 3.83 . The heavy lines in Fig. 2(b) show the measured emission from QCL1 after introduction of the ADFB grating. Note that $\rho(f)$ is symmetric about $f_{\mathrm{B}}$, and the choice of $\Lambda$ places $f_{\mathrm{B}}$ between the two gain maxima. In contrast to the multi-mode FP emission, ADFB-controlled emission tends to occur on only two dominant lasing modes for any given operating bias, each mode falling close to a single gain maxima. With increasing QCL electrical bias the effective gain maxima migrate, and the dual-mode emission switches between individual ADFB bands following the effective gain movement. Such highly selective mode switching behaviour is in fact only possible in the presence of the changing spectral gain, as explained using a full time-domain model in Ref. 25. It is therefore possible to achieve high side-mode suppression ratios (SMSR) for ADFB controlled modes. Figure 2(e) contains selected emission spectra from QCL1, plotted on a logarithmic intensity scale, showing ADFB modes with an SMSR $>20 \mathrm{~dB}$. More generally, the SMSR values vary for each mode pair but usually exceed $10 \mathrm{~dB}$. Figures 2 (f) and 2(g) show the calculated $\rho(f)$ for a second device (QCL2, $\Lambda=14.60 \mu \mathrm{m}, f_{\mathrm{B}}=2.79 \mathrm{THz}$ ) and its measured emission spectra, respectively. Qualitatively similar behaviour to QCL1 is observed, with single mode lasing under each gain maxima. The $60 \mathrm{GHz}$ offset in $f_{\mathrm{B}}$ compared to QCL1 is presumed to have little impact on laser behaviour, as the average ADFB band spacing and strength remain unchanged. However, QCL2 was processed from a different part of the MBE grown wafer and displays slightly different gain behaviour; with reduced tuning of the lower frequency effective gain maxima. Consequently, the low frequency ADFB controlled mode does not switch between ADFB bands.

Measured electrical $(V-J)$ and output power $(L-J)$ characteristics for QCL1, QCL2, and a third device (QCL3, $\Lambda=14.60 \mu \mathrm{m}, f_{\mathrm{B}}=2.79 \mathrm{THz}$ ) are presented in Fig. 3(a). Introduction of the ADFB grating increases laser threshold current densities by $\sim 10 \%$. As a consequence, an ADFB QCL has higher operating bias than in its original FP state at the same driving current density $(J)$. For a given $J$, gain narrowing is therefore comparatively larger in the ADFB QCLs,
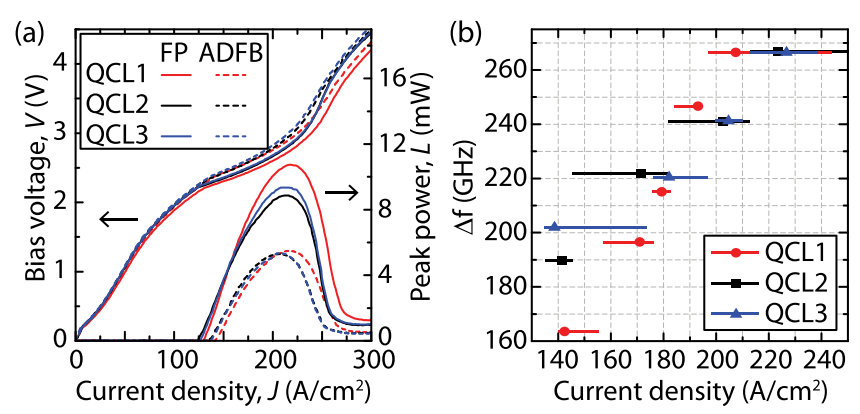

FIG. 3. (a) Electrical and THz output power behaviour of the QCLs before (solid lines) and after (dashed lines) introduction of ADFB gratings. (b) Frequency separation and driving current ranges (horizontal lines) of the dual lasing modes in three ADFB-modified QCLs. Optimum SMSR for each lasing pair was achieved at the points indicated by the symbols (circles, squares, triangles). and the two effective gain maxima are more pronounced. After ADFB introduction, maximum QCL output powers are also reduced by approximately a factor of two. This reduced power and the increase in laser threshold stem from the additional scattering losses associated with the FIB implementation of the ADFB grating, estimated to be $<1 \mathrm{~cm}^{-1}$. Note that the maximum operating temperature of the QCLs also fell by approximately $10 \mathrm{~K}$ after FIB milling, from $\sim 68$ to $\sim 58 \mathrm{~K}$. Dual-mode emission was maintained until close to the maximum operating temperature.

Switchable, dual-mode emission allows one to quantify a varying frequency separation between the two lasing modes, $\Delta f$ (see Fig. 2(b)). For QCL1, five distinct $\Delta f$ values are possible, ranging from 163 to $267 \mathrm{GHz}$. These values are presented in Fig. 3(b), along with the driving current conditions under which they are achieved (horizontal lines). The driving current densities at which the best SMSR were observed are indicated by the circles. Figure 3(b) also shows $\Delta f$ and driving current ranges for QCL2 and QCL3.

Finally, further to mode switching, lasing frequencies (and hence $\Delta f$ ) can be fine-tuned continuously over a short range either electrically (i.e., with driving current or voltage) or via operating temperature $(T)$. As an example, Fig. 4(a)
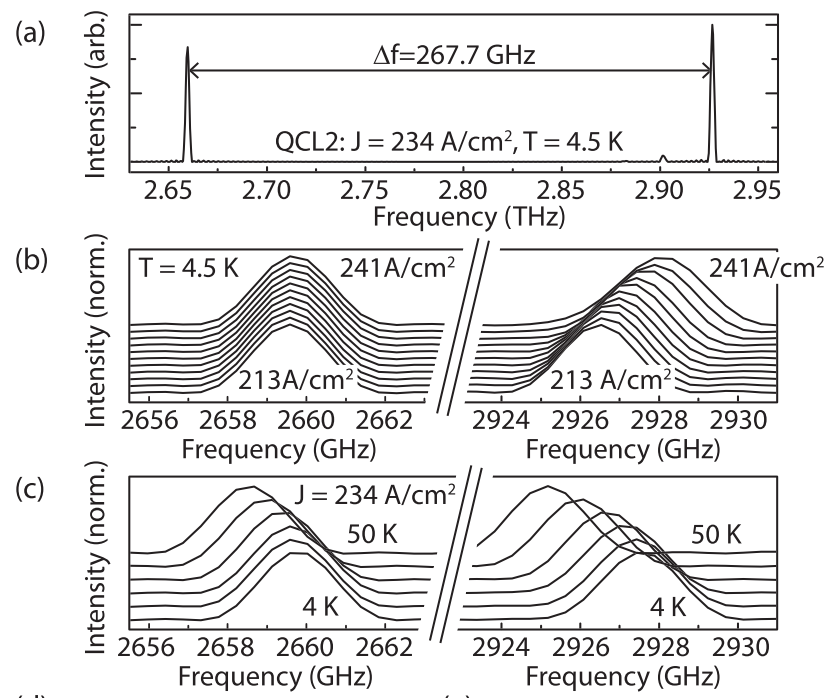

(d)

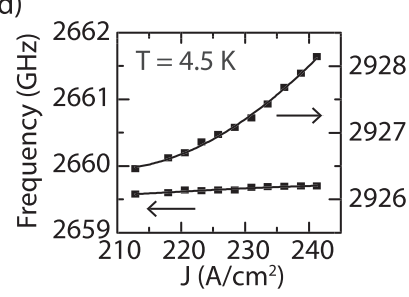

(e)

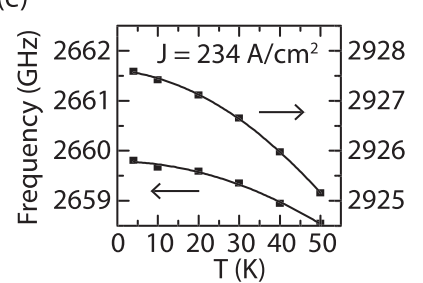

(f)

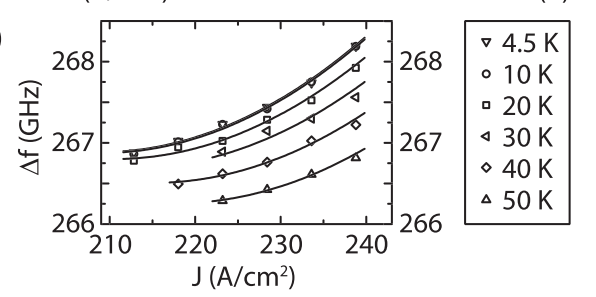

FIG. 4. (a) Selected dual-mode emission spectra from QCL2. Fine tuning of each mode with (b) driving current density and (c) heatsink temperature. For clarity, the mode centre frequencies from (b) and (c) are plotted in (d) and (e), respectively. (f) Variation of both driving current and temperature allows continuous $\Delta f$ tuning of $\sim 2 \mathrm{GHz}$. 
shows one ADFB controlled mode pair from QCL2 at $J=234 \mathrm{~A} / \mathrm{cm}^{2}$ and $T=4.5 \mathrm{~K}$. Lasing occurs simultaneously at 2.66 and $2.93 \mathrm{THz}$. Figures 4(b) and 4(c) show fine tuning of these two lasing modes by independently varying $J$ and $T$, respectively. For clarity, the measured mode centre frequencies are plotted in Figs. 4(d) and 4(e). Under driving current variation the lower frequency mode tuning is negligible $(<100 \mathrm{MHz})$, whereas the higher frequency mode experiences a blue-shift of over $1.5 \mathrm{GHz}$. On the other hand, both modes display a red-shift of between 1.5 and $2.5 \mathrm{GHz}$ as $T$ is increased from 4.5 to $50 \mathrm{~K}$. The disparity in tuning range and direction suggest that two different mechanisms are responsible; increased driving current is not simply heating the laser (i.e., Joule heating). Electrical fine tuning is attributed to mode pulling in a laser system in which the effective gain spectrum varies with driving current. The multi-peaked and changeable gain shape, along with the nonlinear dispersion of the ADFB spectral response, ${ }^{25}$ also explain why we see varying mode pulling rates, ranges, and directions amongst the lasing modes. Temperature tuning is more predictable, each mode displaying a roughly comparable red-shift. This is attributed to the temperature dependence of the refractive index of GaAs, ${ }^{19}$ as thermal expansion of GaAs is negligible in this temperature range. ${ }^{27}$ The individual mode tuning in Figs. 4(d) and 4(e) give rise to continuous changes in $\Delta f$. Figure 4(f) shows the achievable $\Delta f$ for this mode pair; appropriate choices of $J$ and $T$ allow $\Delta f$ to be placed within a $\sim 2 \mathrm{GHz}$ span around $267.5 \mathrm{GHz}$.

In conclusion, ADFB gratings were applied to heterogeneous $\mathrm{THz}$ QCLs with dual-peaked gain spectra. The ADFB gratings generate multi-band filtering for both gain maxima, resulting in simultaneous dual-mode lasing. Discrete electronic mode tuning between ADFB bands produces a variation in the dual-mode separation of $>100 \mathrm{GHz}$, with continuous electronic and thermal fine tuning of up to $2 \mathrm{GHz}$. Such devices might be beneficial where tunable dual-mode emission is desirable, for example, in difference frequency generation schemes, as only a single source must be stabilized. More generally, this result demonstrates the capability of short, holographically designed ADFB gratings providing multi-frequency optical filtering over a broad spectral range.

This work was supported by EPSRC First Grant EP/G064504/1 and partly supported by HMGCC.
${ }^{1}$ R. Köhler, A. Tredicucci, F. Beltram, H. E. Beere, E. H. Linfield, A. G. Davies, D. A. Ritchie, R. C. Iotti, and F. Rossi, Nature (London) 417, 156-159 (2002)

${ }^{2}$ B. S. Williams, Nat. Photonics 1, 517-525 (2007).

${ }^{3}$ M. Tonouchi, Nat. Photonics 1, 97-105 (2007).

${ }^{4}$ M. I. Amanti, G. Scalari, R. Terazzi, M. Fischer, M. Beck, J. Faist, A. Rudra, P. Gallo, and E. Kapon, New J. Phys. 11, 125022 (2009).

${ }^{5}$ G. Scalari, M. I. Amanti, C. Walther, R. Terazzi, M. Beck, and J. Faist, Opt. Express 18, 8043-8052 (2010).

${ }^{6}$ J. R. Freeman, O. P. Marshall, H. E. Beere, and D. A. Ritchie, Opt. Express 16, 19830-19835 (2008).

${ }^{7}$ S. P. Khanna, M. Salih, P. Dean, A. G. Davies, and E. H. Linfield, Appl. Phys. Lett. 95, 181101 (2009).

${ }^{8}$ D. Turčinková, G. Scalari, F. Castellano, M. I. Amanti, M. Beck, and J. Faist, Appl. Phys. Lett. 99, 191104 (2011).

${ }^{9}$ J. R. Freeman, A. Brewer, J. Madéo, P. Cavalié, S. S. Dhillon, J. Tignon, H. E. Beere, and D. A. Ritchie, Appl. Phys. Lett. 99, 241108 (2011).

${ }^{10}$ C. Gmachl, D. L. Sivco, R. Colombelli, F. Capasso, and A. Y. Cho, Nature (London) 415, 883-887 (2002).

${ }^{11}$ G. Scalari, C. Walther, J. Faist, H. Beere, and D. Ritchie, Appl. Phys. Lett. 88, 141102 (2006).

${ }^{12}$ L. Lever, N. M. Hinchcliffe, S. P. Khanna, P. Dean, Z. Ikonić, C. A. Evans, A. G. Davies, P. Harrison, E. H. Linfield, and R. W. Kelsall, Opt. Express 17, 19926-19932 (2009).

${ }^{13}$ J. Xu, J. M. Hensley, D. B. Fenner, R. P. Green, L. Mahler, A. Tredicucci, M. G. Allen, F. Beltram, H. E. Beere, and D. A. Ritchie, Appl. Phys. Lett. 91, 121104 (2007).

${ }^{14}$ A. W. M. Lee, B. S. Williams, S. Kumar, Q. Hu, and J. L. Reno, Opt. Lett. 35, 910-912 (2010).

${ }^{15}$ Q. Qin, J. L. Reno, and Q. Hu, Opt. Lett. 36, 692-694 (2011).

${ }^{16}$ L. Mahler, A. Tredicucci, R. Köhler, F. Beltram, H. E. Beere, E. H. Linfield, and D. A. Ritchie, Appl. Phys. Lett. 87, 181101 (2005).

${ }^{17}$ B. S. Williams, S. Kumar, Q. Hu, and J. L. Reno, Opt. Lett. 30, 2909-2911 (2005).

${ }^{18}$ S. Chakraborty, T. Chakraborty, S. P. Khanna, E. H. Linfield, A. G. Davies, J. Fowler, C. H. Worrall, H. E. Beere, and D. A. Ritchie, Electron. Lett. 42, 404-405 (2006).

${ }^{19}$ M. S. Vitiello and A. Tredicucci, IEEE Trans. Terahertz Sci. Tech. 1, 76-84 (2011).

${ }^{20}$ A. Straub, C. Gmachl, D. L. Sivco, A. M. Sergent, F. Capasso, and A. Y. Cho, Electron. Lett. 38, 565-567 (2002).

${ }^{21}$ L. Mahler, A. Tredicucci, F. Beltram, C. Walther, J. Faist, H. E. Beere, D. A. Ritchie, and D. S. Wiersma, Nat. Photonics 4, 165-169 (2010).

${ }^{22}$ R. Blanchard, S. Menzel, C. Pflügl, L. Diehl, C. Wang, Y. Huang, J.-H. Ryou, R. D. Dupuis, L. D. Negro, and F. Capasso, New J. Phys. 13, 113023 (2011).

${ }^{23}$ S. Chakraborty, M. C. Parker, and R. J. Mears, Photonics Nanostruct. Fundam. Appl. 3, 139-147 (2005).

${ }^{24}$ S. Chakraborty, O. P. Marshall, M. Khairuzzaman, C.-W. Hsin, H. E. Beere, and D. A. Ritchie, Appl. Phys. Lett. 101, 121103 (2012).

${ }^{25}$ S. Chakraborty, O. P. Marshall, C.-W. Hsin, M. Khairuzzaman, H. E. Beere, and D. A. Ritchie, Opt. Express 20, B306-B314 (2012).

${ }^{26}$ N. Jukam, S. S. Dhillon, D. Oustinov, Z.-Y. Zhao, S. Hameau, J. Tignon, S. Barbieri, A. Vasanelli, P. Filloux, C. Sirtori, and X. Marcadet, Appl. Phys. Lett. 93, 101115 (2008).

${ }^{27}$ T. Soma, J. Satoh, and H. Matsuo, Solid State Commun. 42, 889-892 (1982). 